\title{
Research on Raman Enhancement of Surface Materials Based on Biological Sensors
}

\author{
Mayilamu Musideke ${ }^{1, a}$, Shentong $\mathrm{Mo}^{2, \mathrm{~b}}$, Qiang Zou ${ }^{2, \mathrm{c}}$, \\ Aihaiti Rouzi ${ }^{1, a}$ and Wenxiao Zeng ${ }^{3, a}$ \\ ${ }^{1}$ Xinjiang Uygur Autonomous Region Fiber Inspection Bureau, New Urban District, Urumqi, \\ 830011, P. R. China \\ ${ }^{2}$ School of electronic and information engineering, Tianjin University, Nankai District, Tianjin, \\ 300072, P. R. China \\ ${ }^{3}$ Xinjiang Infotool CO.,LTD, 2nd Science Street, Urumqi, 830011, P. R. China \\ amahiralaser@163.com, ${ }^{\mathrm{b}}$ moshentong678@126.com, ${ }^{\mathrm{c} z o u q i a n g @ t j u . e d u . c n}$
}

Keywords: Biological sensor; Ion sputtering coating; Raman spectrum; Surface Enhanced Raman Spectroscopy; Scanning electron microscope

\begin{abstract}
Optical fiber sensing technology has been shown to advantage in physical testing. In terms of chemical content and biomass detection, its specificity and flexibility cannot meet the demand of the rapid development of biology, chemistry, due to the material, structure and process of bondage. At the same time, along with the progress of nanometer materials preparation and testing technology, nanotechnology applications become mature in the field of biochemical. This paper introduces the development status of fiber optic sensor and the Raman spectra of metal nanoparticles. The various cases of Raman spectroscopy and scanning electron microscope were mainly introduced in the paper, compared with sample under different conditions. By observing the surface morphology and composition of samples, and understanding the generation mechanism of samples, analyzing the effect of a variety of experimental conditions on the generated samples, the optimization of conditions was selected.
\end{abstract}

\section{Introduction}

In the US, GE carries on bionics researches in nanotech and develops a new generation of biosensors to achieve rapid detection of various biological threats, funded by the DARPA [1,2]. In Europe, the SPOT-NOSED(Single Protein Nano-biosensor Grid Array) Project combines information science and nanoscale materials and makes a breakthrough in the development of nano-biosensors based on individual olfactory receptors, supported by the FET, a subsidiary of the European Commission. In China, the sensing and detection technology of chemical and biological information is also one of the key research directions all the time, and it has many important practical applications in health, environment and security areas [3-5]. The outline of the national long and medium-term program for scientific and technological development (2006-2020) emphasizes that, to strengthen the researches in basic science and frontier technology, especially in interdisciplinary sciences, is a focal point of the development of science and technology [6-7].

Started by the measurement of the $\mathrm{pH}$ value at the beginning of the last century in 1906, biochemical sensors have undergone several eras since its emergence. Though its testing means developed from traditional biochemical reagents to modern detection methods in physics such as various optical, electric and magnetic means, it is always the joint production of many different subjects [8-10]. And nowadays, as the biology and chemistry science gain more and more national attention all over the world, the researches of biochemical sensors are becoming the hot fields chased by scientists. 
Until now, the biochemical sensors are usually classified into three types based on the different transfer methods: electrochemical type, thermochemical type and photochemical type, etc. And the photochemical type can be subdivided into optical methods, spectral methods and optical fiber. Fiber-optic biosensors(FOBS), as an integration of optical fiber sensing technology and modern biochemical detection methods, are always at the cutting-edge of the development of the biochemical sensor technology [11-13].

With the development of fiber-optic biosensors, combined with the efficient scattering reflection of the properties of optical fiber's microstructure, the utilization of the optical and spectroscopic techniques provides a means for the detection and analysis of biochemical signals [14-15]. For example, the application of a large number of the analysis methods for optical background in the detection process of FOBS can realize remote distributed measurement, and with the applied technology of Wavelength Division Multiplexing-WDM, FOBS offers the possibility to the analysis of multi-targets based on a single sensor.

However, there also exists some problems to be resolved during the development of FOBS. Restricted by the materials, structures and technics, FOBS have little relation with the chemical or biological state of the samples, as a result, with its high specificity and poor flexibility, FOBS can hardly meet the demand of the rapid development of biochemical industry so that its application range is limited [16]. Meanwhile, the application of nano technology in biochemical sensing field both theoretically and practically is getting mature, which has an edge in materials and structures. In conjunction with researches on microstructures in optical fibers doped with nano materials, it is expected to improve traditional fiber-optic sensing technology to expand the application range of FOBS in biological detection.

\section{Experimental Methods}

2.1 Experimental Scheme. In recent months, we carried out surface modification treatment on the substrates of different materials in various ways based on our laboratory concrete conditions and then conducted SERS measurement on these fiber-optical samples. In each method we designed several nanostructures of metal particles under different conditions, made comparison of their different SEM photos, and observed their surface morphology and compositions, as well as studied their formation mechanism; meanwhile, we studied the SERS spectra of different samples to analysis the influence of these experimental conditions on samples, so that we could find out the optimal preparation project on fiber-optical biosensors in laboratory.

Hydrofluoric acid (HF) is commonly used in removing the coat layer of the glass-coated microwires, and it can remove the glass coated layer of $10 \mu \mathrm{m}$ thickness within 150 seconds. Moreover, the compositions and structures of the optical fibers surface are the important factors of the SERS detection performance of FOBS. So, considering the influences of the corrosion concentration, corrosion time of HF and the disposition time of metal particles on the SERS detection of FOBS, we made three kinds of substrates with different materials: glass, quartz and solid-core fibers, and conducted the same treatment on them as follows.

According to orthogonal experimental method, we prepared several experiments on the silver plated samples with a series of gradient conditions, evaluated the corrosion behaviors of HF in the surface treatments of the optical fibers materials, analyzed SERS performances of the samples under different corrosion behaviors and explored their mechanism of surface enhanced Raman scattering.

2.2 Orthogonal experiments on glass sheets. Screen out the optimal surface treatment project through a series of orthogonal experiments. Firstly, set up different HF concentration gradient (5\%, $10 \%, 15 \%, 20 \%, 25 \%, 30 \%$ ), then process the sheets in the same way. Secondly, set up different HF corrosion time gradient (5min, 10min, 15min, 20min, 25min, 30min), then process the sheets in the same way. Finally, Set up different metal particle disposition rate, that is, samples with coating layers of different thicknesses $(20 \mathrm{~nm}, 40 \mathrm{~nm})$, then process the sheets in the same way. 
From the experiments above, we can find out the optimal conditions, which are suitable for many kinds of detection, such as crystal violet, rhodamine and methylene blue.

2.3 Orthogonal experiments on quartz sheets. To explore the optimization of quartz component and glass component on SERS, based on the previous experiments on glass sheets, design two groups of experiments as follows: Group One: Set up different HF concentration gradient $(10 \%, 20 \%, 30 \%$, $40 \%$ ), then process the sheets in the same way. Group Two: Set up different HF corrosion time gradient (30min, 60min), then process the sheets in the same way.

2.4 Experiments on optical fibers. At last, based on the orthogonal experiments on glass sheets and quartz sheets, we have already got the optimal corrosion concentration, corrosion time and metal particle disposition rate (coating thickness), and now we can apply the conditions to optical fibers to obtain the best enhancement in SERS.

Firstly, coat the surface of solid-core fibers with a layer of silver particle of $20 \sim 40 \mathrm{~nm}$ thickness; coat the inner wall of hollow fibers with a layer of silver colloid. Secondly take out large sample accessories from the Laser Roman Spectrometer and directly put the testing fiber probe into the solution to be tested, such as dye molecules like crystal violet, rhodamine and methylene blue, regarding optical fibers as the active sources. Then detect the samples with the 533/632nm laser. Finally, after a series of detection of samples, select out the optimal fiber-optical sensors and apply it to the biological detection.

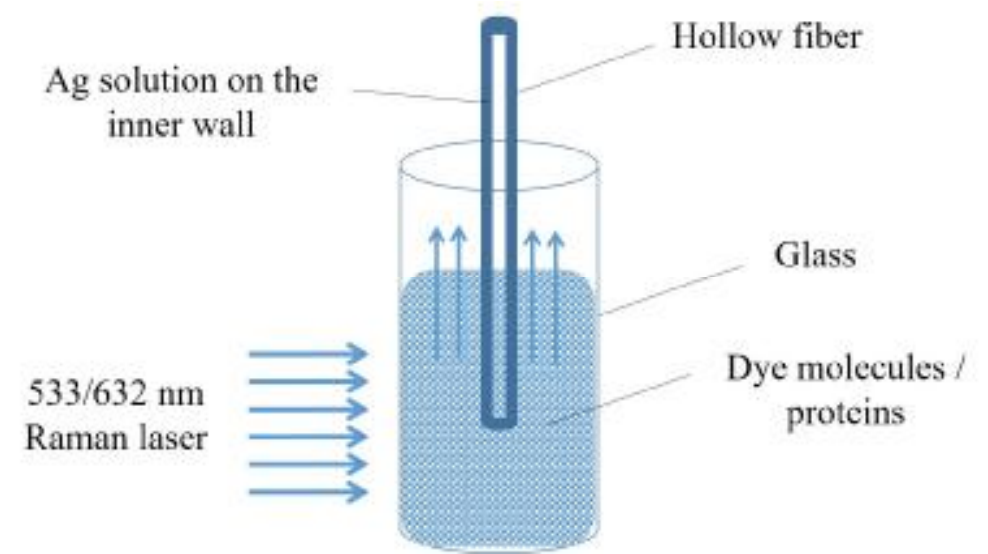

Fig.1 Process diagram of optical fiber sensor surface treatment

\section{Results and Discussion}

3.1 Analysis of concentration gradients. Through the above-mentioned sheet glass and quartz fiber series of experiments and orthogonal experiments, we can have the following Raman spectra. We have to choose the most optimal corrosion treatment solution for surface modification of fiber optic sensor material, thereby improving the performance of the optical fiber sensor.
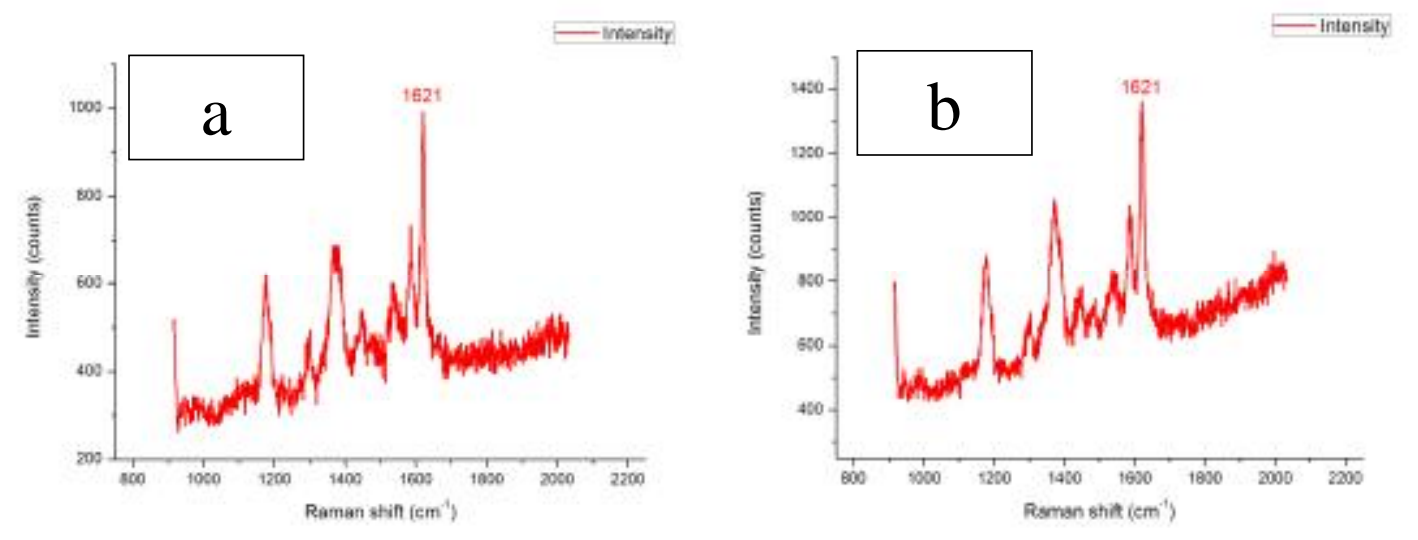

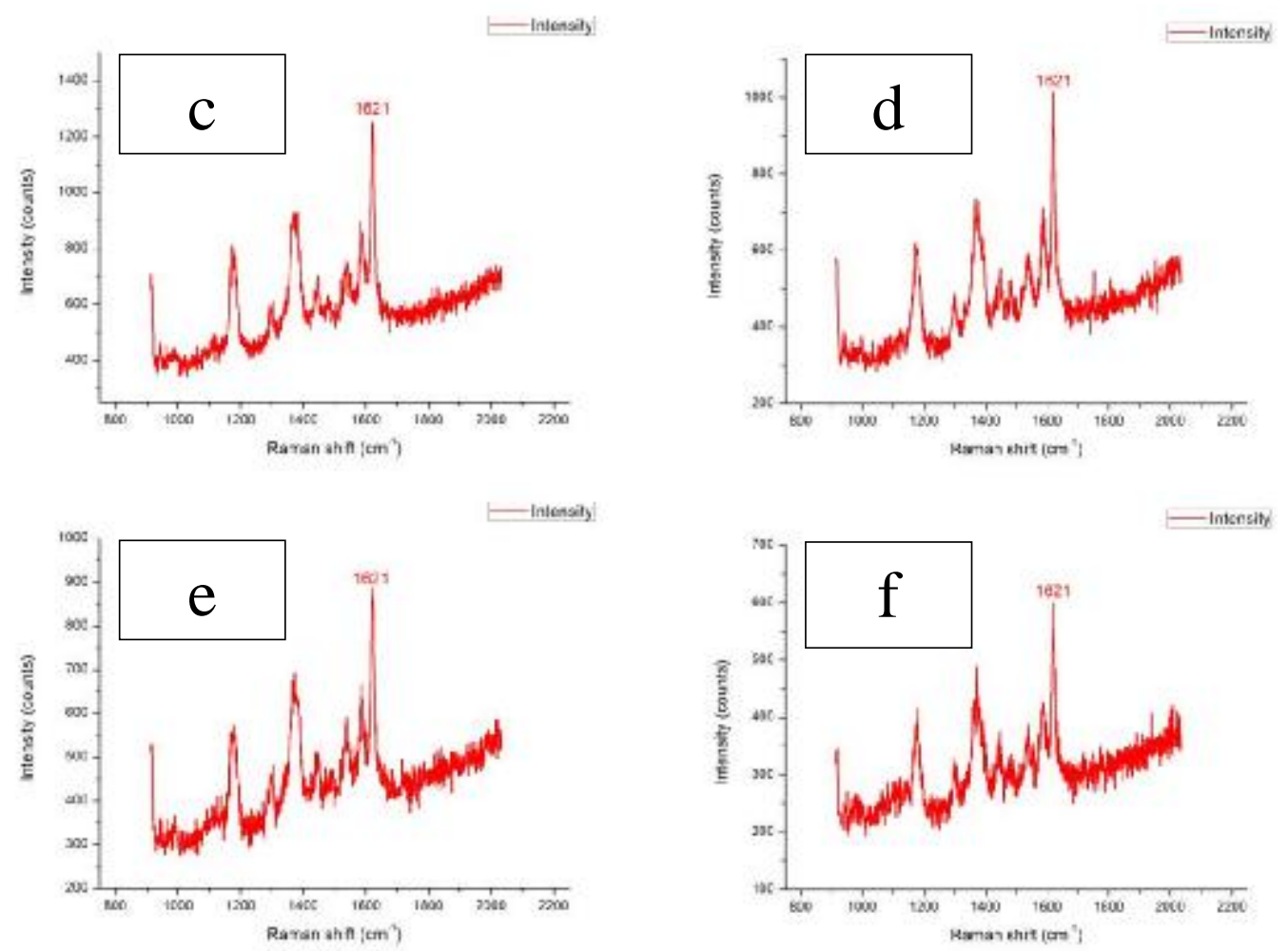

Fig.2 With $20 \mathrm{~nm}$ Au film, concentration of corrosion and time is respectively: (a)5\%:5 min; (b)10\%:5 min; (c)15\%:5 min; (d)20\%:5 min; (e) $25 \%: 5 \mathrm{~min}$; (f) $30 \%: 5 \mathrm{~min}$, on the Raman spectra of glass

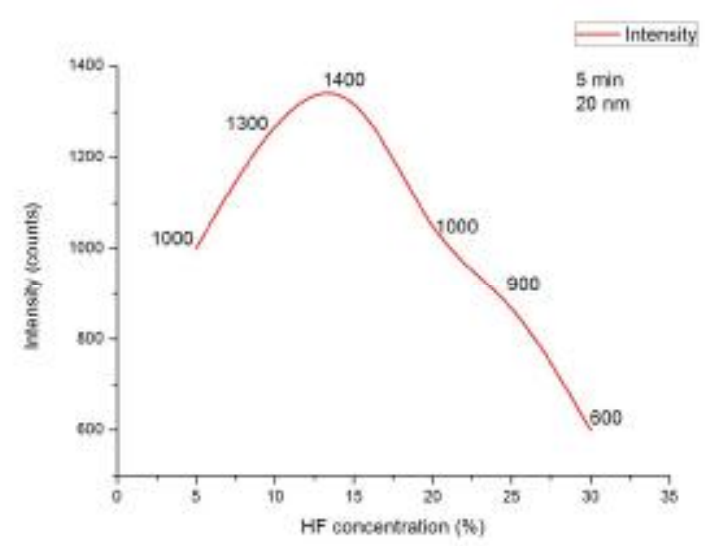

Fig.3 Relationship between the surface enhanced Raman intensity of the glass sheet and the corrosion concentration of HF acid, when the time of corrosion is $5 \mathrm{~min}$ and the thickness of Au film is $20 \mathrm{~nm}$.

As can be seen from Fig.2 and Fig.3, surface Raman enhancement factor will first rise and fall with increasing concentration of corrosion.

3.2 Analysis of time gradients. As can be seen from the Fig.4, surface Raman enhancement factor will first rise and fall with increasing the corrosion of time. When the HF acid etching time is about 15 min, Raman enhancement factor reaches its maximum, and fiber glass surface morphology processing to achieve the best results. At the same time, in a certain concentration of HF acid etching time and etching concentration, Raman enhancement factor with $20 \mathrm{~nm}$ Au film is larger than which is plated $40 \mathrm{~nm}$ film. So glass or fiber surface topography treatment can achieve better results. 

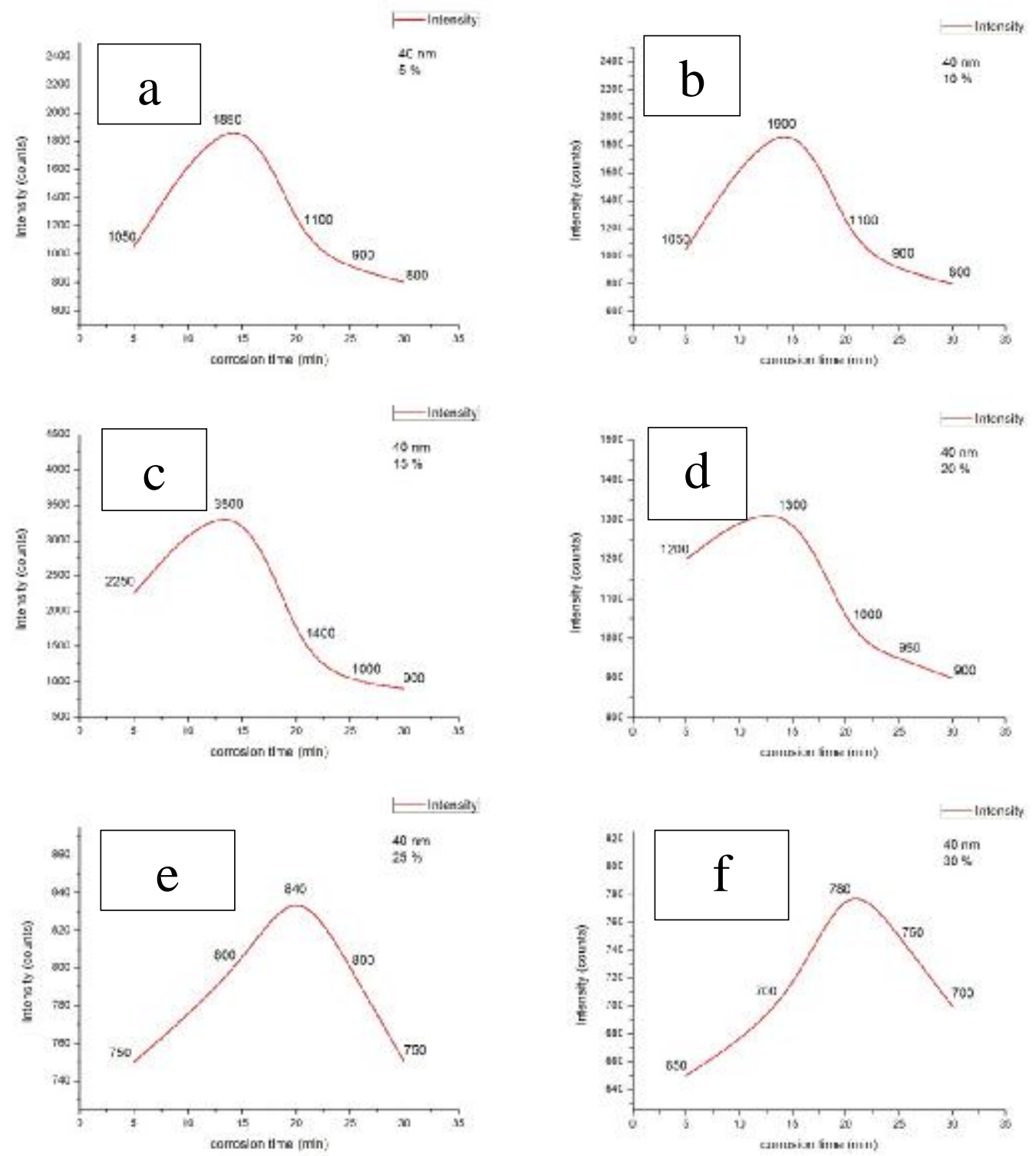

Fig.4 With $40 \mathrm{~nm}$ Au film, the concentration of the corrosion is respectively: (a) $5 \%$; (b) $10 \%$; (c) $15 \%$; (d) $20 \%$; (e) $25 \%$; (f) $30 \%$

Relationship between the surface enhanced Raman intensity of the glass sheet and the corrosion time of HF acid.

For quartz substrate, in the same manner, surface Raman enhancement factor will first rise and fall with increasing the corrosion of time and the concentration of corrosion. When the HF concentration of $10-15 \%$ acid corrosion, etching time is about $15 \mathrm{~min}$, the Raman surface enhancement factor reaches its maximum. It can make the surface morphology processing to achieve better results.

At a certain concentration of HF acid etching and etching time, quartz, with the same kinds of plating thickness of the Au film, has big surface Raman enhancement factor. It can make the optical fiber sensor surface morphology processing to achieve better results. 

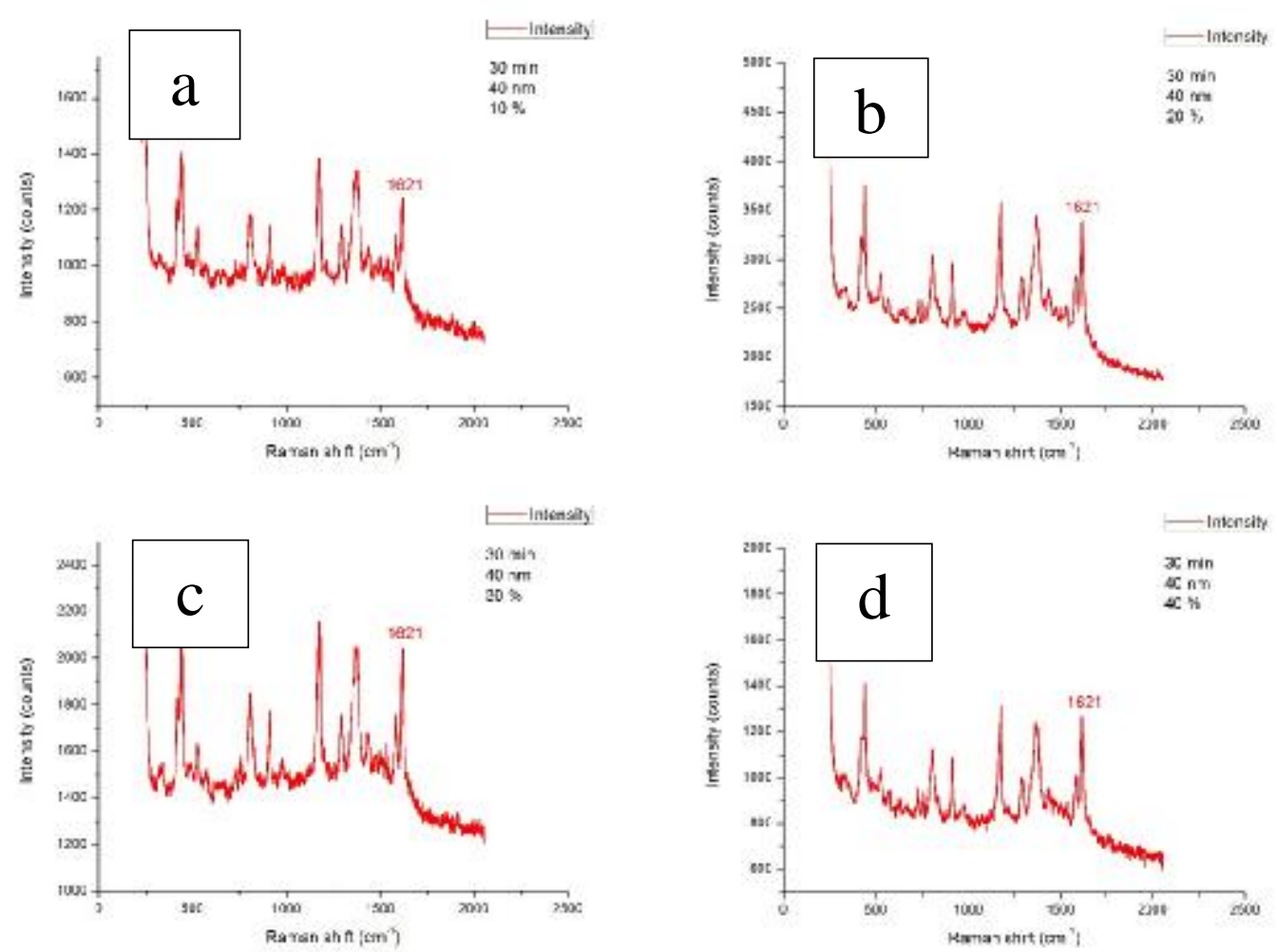

Fig.5 With $40 \mathrm{~nm}$ Au film, the concentration of the corrosion is respectively: (a)10\%: $30 \mathrm{~min}$; (b)20\%:30 min; (c)30\%:30 min; (d) $40 \%: 30$ min, on the quartz piece of Raman spectra.

3.3 Analysis of SEM images. According to the analysis of Raman spectra, film coated quartz surface-enhanced Raman factor is much larger than the glass under the same conditions. To further explore the surface morphology of the quartz with being treated. Two quartz plates were carried out SEM testing, done in an SEM image. The effect of the image whose testing time is 30 min is more obvious and easier to analyzed. The group of samples SEM image are took when the electron microscope is at a magnification of 2000.
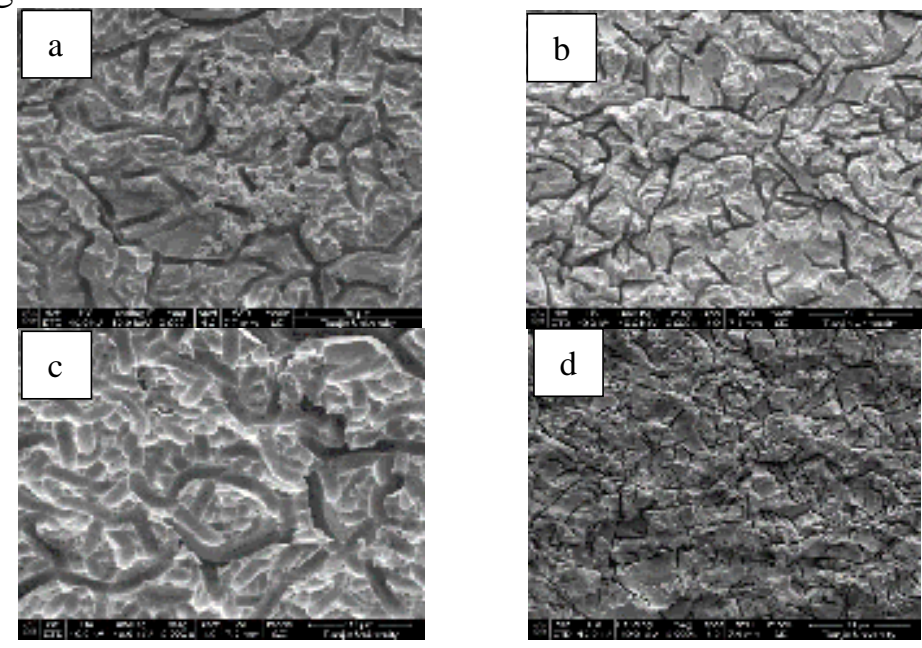

Fig. 6 With $40 \mathrm{~nm}$ Au film, the concentration of the corrosion is respectively: (a)10\%:30 min; (b)20\%:30 min; (c)30\%:30 min; (d) $40 \%: 30 \mathrm{~min}$, the electron microscope is at a magnification of 2000.

As can be seen in Fig.6, the c sample and d sample had some bud shape bodies, but did not grow as the nanowire and nano projections. Because it may be corrosion by high concentration. And although b sample grows out of the nanowires, but its quantity is not enough. The effect is not good enough. Relatively, a sample effect is good, but the length of the nanowires is insufficient ideal. The effect will be better with higher concentration. 
The experiment took easily ion sputtering method. On the one hand, this kind of coating method has good ease of use, simple structure, good quality; On the other hand, Au outermost electron has the whole structure, and it has stable structure which is used to the surface of the sensor, so that it can have stable performance. This method, simultaneous roughening treatment, and the Au plating target can improve adhesion molecules to the sensor surface, thereby enhancing the Raman detection signal molecules, improving optical fiber sensor sensitivity.

\section{Conclusions}

Hydrofluoric acid corrosion is an important means of roughness processing, it is easy to react with glass, quartz material, which treated after it surface can generate nanometer protuberant. In that it can enhance the Raman signal detection, improve the sensitivity of the sensor. The key of this means is how to control the concentration of hydrofluoric acid corrosion and the corrosion time, thereby it can make optical fiber sensor SERS Raman detection sensitivity achieve the best effect.

This paper adopts the method of the ion sputtering coating, this coating method is of simple structure, with good quality and easy to use. Au is a stable metal, and it has stable chemical properties. It not easy to be oxidized because the outermost electron structure is complete. It is plated on the sensor surface, which can form a stable structure. The fiber optic sensor which plated by Au, improves the adhesion molecules on the surface of the sensor, and enhances SERS Raman signal detection, so that it improves the sensitivity of optical fiber sensor. However, this highly enhanced Raman signal cannot be simply attributed to glass or quartz surface roughness handling and Au coating adsorption after an increasing in the number of molecules, and there must be some kind of physical effect at work. This is called the Raman effect which is similar to the X-ray scattering and the Compton effect. The frequency of the light will change after scattering. For materials in glass or quartz substrate, likewise, surface enhanced Raman factor with the increase of concentration of corrosion and corrosion time, decreasing after increasing, when the concentration of $\mathrm{HF}$ acid corrosion in $10 \sim 15 \%$, the corrosion time within 15 min, surface enhanced Raman factor reaches the maximum, the surface morphology of processing to achieve the best effect.

Under certain concentration of hydrofluoric acid corrosion and the corrosion time, with the same thickness of Au plating film, quartz material surface enhanced Raman factor is bigger than glass material, and the processing of surface morphology of fiber optic sensor can achieve better results. Our fiber optic sensor material using quartz material effect will be better. Raman signal is stronger, and with higher sensitivity.

\section{Acknowledgements}

This work was financially supported by Science and Technology Support Project of Science and Technology Office in Xin Jiang (2015).

\section{References}

[1] Wang S X,Li G X. IEEE TRANSACTIONS ON MAGNETICS, 44(7): 1687-1702 (2008).

[2] Shradha Prabhulkar, Li C Z. In: 3rd International Conference on Bioinformatics and Biomedical Engineering. Beijing, China:1-4 (2009).

[3] Luis P. Fonseca, Verónica C.B. Martins, Paulo P. Freitas. In: 1st Portuguese Meeting in Bioengineering. Lisbon, Portugal:1-4 (2011).

[4] Tessy Theres Baby, Sundara Ramaprabhu. In: International Conference on Nanoscience, Technology and Societal Implications. Bhubaneswar, India:1-6 (2011).

[5] Ahmet A. Yanik, Min Huang, Alp Artar. In: 23rd Annual Meeting of the IEEE Photonics Society. Denver CO, USA:162-163 (2010). 
[6] Vikram Krishnamurthy, Kai Yiu Luk, Bruce Cornell. In: IEEE International Conference on Acoustics, Speech and Signal Processing. Honolulu, Hawaii, USA: I-401-I-404 (2007).

[7] P. Palestri, R. Sette, F. Pittino. In: 14th International Conference on Ultimate Integration on Silicon. Warwick, United Kingdom:77-80(2013).

[8] Sahar M.Monfared, Vikram Krishnamurthy, Bruce Cornell. In: IEEE International Workshop on Genomic Signal Processing and Statistics. Minneapolis, MN, USA: 1-6 (2009).

[9] C. Buchheim, G. Kittler, V. Cimalla. In: Proceedings of IEEE Sensors. Vienna, Austria: . 1007-1010 (2004).

[10] Li P, Jing G S, Cui T H. In: IEEE SENSORS. Valencia, Spain: 873-876 (2014).

[11] David E. Arthur, Eddy O. Nnabuk, Casimir E. Gimba. International Research Journal of Pure and Applied Chemistry, 6(4), 160-173(2015).

[12] T.R.M. De Beer, P. Vercruysse, A. Burggraeve et al. Journal of Pharmaceutical Sciences, 98 (9), 3430-3446 (2009).

[13] Sitansu Sekhar Nanda, Min Jik Kim, Kwi Seok Yeom et al. Trends in Analytical Chemistry, 80, 125-131 (2016).

[14] Zai Qing Wen. Journal of Pharmaceutical Sciences, 6 (11), 2861-2878 (2007).

[15] Zhe Xing, Changwen Du, Kang Tian et al. Talanta, 158, 262-269 (2016).

[16] O. Unsalan, A.H. Kuzucuoglu. Spectrochimica Acta Part A: Molecular and Biomolecular Spectroscopy, 152, 572-576 (2016). 